\title{
Visitantes florales y potencial polinizador de trichocentrum stramineum orquídea amenazada y endémica de Veracruz
}

Floral visitors and potential pollinator of trichocentrum stramineum endangered and endemic orchid of Veracruz
Saúl Hernández Carmona ${ }^{1}$, Gustavo Carmona-Díaz ${ }^{1,2}$, Paulo Cesar Quintana Morales ${ }^{1}$, Jorge E. Morales ${ }^{1}$, Alejandro Retureta Aponte ${ }^{2}$ y Victor Rico-Gray ${ }^{1}$.
${ }^{1}$ Instituto de Neuroetología, Universidad Veracruzana, Luís Castelazo Ayala s/n, Col. Industrial Animas, C.P. 91190. Xalapa, Veracruz, México.

${ }^{2}$ Facultad de Ingeniería en Sistemas de Producción Agropecuaria, Universidad Veracruzana, km 4.5 Carretera Costera del Golfo, Acayucan-Catemaco. C. P. 96000. Acayucan, Veracruz, México.

${ }^{凶}$ Autor para correspondencia: gcarmona@uv.mx

Recibido: 15/07/2017

Aceptado: 10/12/2017

\section{RESUMEN}

Trichocentrum stramineum es una orquídea endémica del centro de Veracruz y en categoría de riesgo como amenazada según la NOM-059-SEMARNAT-2010 por la reducción poblacional donde habita. Poca información se ha generado de esta orquídea en la literatura especializada y hasta antes del presente trabajo se desconocían sus visitantes florales y su potencial polinizador. De 2014 a 2017, se realizaron observaciones directas e indirectas a las inflorescencias de T. stramineum para registrar los visitantes florales y determinar al potencial polinizador en dos localidades de su distribución geográfica. Se registraron dos especies de visitantes florales en la localidad 1 y tres en la localidad 2. La abeja Centris nitida (Apidae) fue observada efectuando la remoción y deposición de los polinios de T. stramineum en ambas localidades. La abeja Centris tarsata (Apidae) fue observada un par de ocasiones en cada localidad sin que realizara remoción ni deposición de los polinios. La avispa Polybia sp., fue observada en dos ocasiones sólo en la localidad 2 sin llevar a cabo la remoción de polinios. La orquídea T. stramineum presenta un sistema especializado de polinización al depender solo de una especie para la fecundación de las flores. Los registros del polinizador de $T$. stramineum son los primeros en obtenerse y documentarse para esta orquídea.

Palabras clave: Centris nitida, Visitante floral, Polinizador, Orquídea.

\begin{abstract}
ABSTRAC
Trichocentrum stramineum is an endemic orchid of Veracruz center and threatened according to the NOM-059-SEMARNAT-2010 for the population reduction where it lives. Little information has been generated from this orchid in the specialized literature and even before the present work; its floral visitors
\end{abstract}


and its pollinator potential were unknown. From 2014 to 2017, direct and indirect observations were made to the inflorescences of $T$. stramineum to register the floral visitors and determine the potential pollinator in two localities of its geographical distribution. Two species of floral visitors were recorded in locality 1 and three in locality 2. The Centris nitida bee (Apidae) was observed carrying out the removal and deposition of the $T$. stramineum pollinia in both locations. The Centris tarsata bee (Apidae) was observed a couple of times in each locality without any removal or deposition of the pollinia. The wasp Polybia sp., was observed twice only in location 2 without carrying out the removal of pollinia. The $T$. stramineum orchid presents a specialized system of pollination by depending only on one species for the fertilization of the flowers. The records of the pollinator of $T$. stramineum are the first to be obtained and documented for this orchid.

Keywords: Centris nitida, Floral visitor, Potential pollinator, Orchid.

\section{INTRODUCCIÓN}

Dentro del grupo de las orquídeas se ha registrado la mayor diversidad de estrategias de polinización, visitantes florales y polinizadores (Tamayo, 2016). Entre los insectos considerados como los más importantes y frecuentes, se encuentran las abejas como las más esenciales para la reproducción de estas plantas (Vinson et al. 1996; Sánchez-Marroquín, 2017). En esta interacción mutualista el polinizador puede llegar a obtener diversas recompensas florales, mientras que la planta asegura la fecundación de sus flores con la remoción y deposición de los polinios (Trejo-Salazar et al. 2015).

La importancia de los polinizadores en Orchidaceae está dada por esta doble visita de remoción y deposición de polinios, desarrollando complejos mecanismos de polinización entre orquídeas e insectos. No obstante, para la mayoría de sus especies se desconocen los potenciales polinizadores por la dificultad de ser vistos y registrados en campo (Torretta et al. 2011). El alto grado de especialización que muchas especies de este grupo de plantas presenta con sus visitantes florales y polinizadores, deriva en mayores retos de estudio, lo que conlleva a que para numerosas orquídeas solo se infiera su mecanismo de polinización efectuada por uno o pocos polinizadores específicos (Damon y Cruz, 2006; Carmona-Díaz y García-Franco, 2009). Por ello, resulta básica la identificación y registro de los polinizadores de muchas orquídeas para entender las estrategias de reproducción de estas especies (Trejo-Salazar et al. 2015).

Trichocentrum es un género polimórfico Neotropical con especies a veces también incluidas en Oncidium y/o Lophiaris, dependiendo del autor, pero de las cuales, más del $80 \%$ de estas se desconocen sus polinizadores, aunque se infiere que son abejas del género Centris (Carmona-Díaz y GarcíaFranco, 2009; Pansarin y Pansarin, 2011). Un ejemplo de esto último es Trichocentrum stramineum descrita originalmente como Oncidium stramineum y que por muchos años se desconoció su potencial polinizador. Trichocentrum stramineum es endémica de Veracruz, México y está catalogada como Amenazada por la NOM-059-SEMARNAT2010 (Soto y Solano, 2007; Flores-Escobar et al. 2008). En el caso particular de T. stramineum no existe información al respecto ni literatura que refiera acerca de las especies que la visitan y/o la polinizan y únicamente se han documentado los siguientes trabajos, "Fichas técnicas de Trichocentrum stramineum" (Soto y Solano, 2007; Moreno-Martínez y Menchaca-García) y "Propagación in vitro de Oncidium stramineum Lindl., una orquídea amenazada y endémica de México" (Flores-Escobar et al. 2008).

Se sabe que debido a la auto-incompatibilidad reproductiva en su sistema de polinización 
muchas especies de los géneros Trichocentrum y Oncidium son polinizadas por abejas solitarias del género Centris (Carmona-Díaz y GarcíaFranco, 2009; Torretta et al. 2011). El género Centris estima unas 232 especies registradas alrededor del mundo y representa una parte de la gran diversidad de abejas conocidas (SánchezMarroquín, 2017). Las abejas Centris puede ser visitantes florales de muchas especies de orquídeas (Liu y Pemberton, 2010); y/o ser los potenciales polinizadores (Pemberton y Liu, 2008). Las abejas Centris al hacer contacto con los polinios, estos se adhieren en su cabeza transportándolos a las flores de otro individuo de la especie (Velez-Velandia, 2012).

El objetivo del presente trabajo fue registrar a los visitantes florales y polinizadores de la orquídea T. stramineum en dos localidades contrastantes de la parte central del estado de Veracruz, identificando a su potencial polinizador por la remoción y deposición de los polinios con la consecuente fecundación de las flores y la producción de frutos.

\section{MATERIALES Y MÉTODOS}

El presente trabajo se desarrolló en dos localidades de la distribución geográfica de la orquídea cuyos nombres y mapa de ubicación se omiten por la situación poblacional crítica que presenta la especie. Ambos sitios están ampliamente separados por barreras naturales que permiten establecer poblaciones naturales de la orquídea (Ver Moreno-Martínez y MenchacaGarcía, 2009). La localidad 1 corresponde a una ecotonía relictual entre selva mediana subcaducifolia y encinar con amplias áreas de vegetación secundaria y cultivo de cafetales. La localidad 2 es un fragmento de selva mediana subperennifolia con encinar tropical asociado a cultivos de limón. Durante cuatro temporadas de floración de $T$. stramineum de 2014 a 2017, en la localidad 1 se realizaron observaciones puntuales directas e indirectas para registrar los visitantes florales y para la determinación del polinizador de la orquídea. En la localidad 2 se efectuaron ambos tipos de observaciones durante tres temporadas de floración de 2015 a 2017.

Las observaciones directas se efectuaron durante la floración pico de abril a mayo. Los registros se enfocaron a cualquier insecto que visitó las inflorescencias de $T$. stramineum y a la actividad de estos durante su interacción con la orquídea. La remoción de polinios de las inflorescencias y la deposición de los mismos en otras flores fueron los indicadores para distinguir entre visitante floral y polinizador. Las observaciones indirectas fueron hechas sobre inflorescencias de T. stramineum que no se usaron para las observaciones directas. Se registró la remoción y/o deposición de polinios, así como el registro de producción de frutos.

\section{RESULTADOS}

Las observaciones directas permitieron registrar dos visitantes florales en la localidad 1 y tres visitantes en la localidad 2 (Cuadro 1). La abeja Centris nitida (Apidae) se registró removiendo y depositando los polinios de T. stramineum (Figura 1). Esta abeja es de tamaño pequeño, tórax claro-amarillo brillante, abdomen negro y clípeo con manchas laterales elipsoidales de color amarillo en las hembras y clípeo amarillo con una mancha basal marrón oscuro y negro en los machos.

En el cuadro 1 se observa que no todos los años de registro fueron observados visitantes florales y la abeja Centris nítida teniendo actividad en las inflorescencias de $T$. stramineum. 


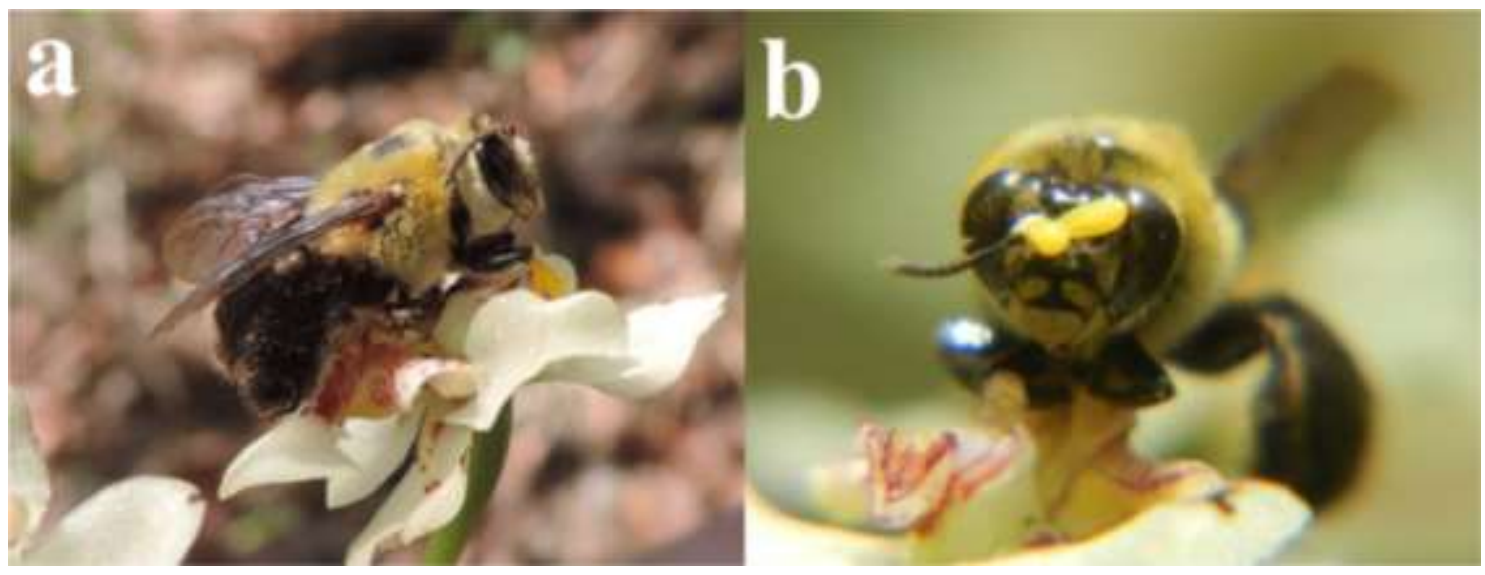

Figura 1. Abeja hembra de Centris nitida. a) de perfil lateral posada en una flor de T. stramineum apreciándose la coloración de su tórax y abdomen. b) con los polinios de T. stramineum impregnados en la parte frontal de su cabeza y parte superior del clípeo.

Cuadro 1. Registro de visitantes florales, remoción de polinios y producción de frutos en T. stramineum durante cuatro periodos de floración en la Localidad 1 y Localidad 2.

Periodos de floración de T. stramineum

$2014 \quad 2016 \quad 2017$

$\begin{array}{llllll}\text { L1 } & \text { L1 } & \text { L2 } & \text { L1 } & \text { L2 } & \text { L1 }\end{array}$

C.n $\quad$ C.n

Visitantes florales observados

* C.t ** ***

C.t ** *

P.sp.

Visitante que removió polinios

$* * *$

C. $n \quad * * * \quad * * *$

C.n

$* * *$

$*$

Producción de frutos

No

$\mathrm{Si} \quad \mathrm{No}$

No

$\mathrm{Si}$

$\mathrm{Si} \quad \mathrm{No}$

Abreviaturas: Localidad 1 (L1); Localidad 2 (L2); Ninguno (*); No se hicieron observaciones (**); No hubo registro (***); Centris nitida (C.n); Centris tarsata (C.t) y Polybia sp. (P. sp.).

Durante las visitas la abeja $C$. nitida interactuó con las flores a través de movimientos rápidos posándose en el centro de la flor en una posición que le permitía hacer contacto con los polinios contenidos en el ginostemo, los cuales se impregnaban en su cabeza efectuando así su remoción y al tomar la misma posición en otra flor, los depositaba en la cavidad estigmática, llevando a cabo el papel de polinizador de la orquídea.

Los otros visitantes florales fueron la abeja Centris tarsata (Apidae) fue observada en las dos localidades en 2015 y 2016 (Cuadro 1) mostrando el mismo patrón de actividad durante 
su interacción con la orquídea. Esta abeja solitaria fue vista un par de ocasiones en cada localidad a través de visitas esporádicas acercándose con movimientos rápidos a las flores, suspendiéndose en el aire y explorándolas por lapsos de dos a tres segundos sin posarse en ellas, retirándose velozmente sin haber tenido contacto con las flores y sin remover los polinios. Otro visitante observado únicamente

\section{DISCUSIÓN}

La orquídea $T$. stramineum presenta un sistema especializado de polinización donde las abejas Centris tienen un papel determinante como polinizadoras de este género de orquídeas. Damon y Cruz (2006), refieren que muchas especies de orquídeas del género Trichocentrum pueden presentar una o pocas especies polinizadoras específicas de abejas Centris. La orquídea $T$. stramineum registró interacción únicamente con tres especies de visitantes florales de los cuales sólo uno mostró un adecuado ajuste morfológico con la flor (Centris nitida), lo que le permitió la remoción y deposición de los polinios.

No obstante, para muchas especies de Orchidaceae y del género Trichocentrum, los estudios de interacción como la polinización son escasos, desconociéndose los sus visitantes florales y/o polinizadores específicos (Pansarin y Pansarin, 2011; Goncalves et al. 2012).

Dentro del contexto de la interacción no sólo las orquídeas presentan sistemas especializados de polinización, sino que muchas abejas del género Centris pueden especializarse en visitar una determinada especie o género de planta, o adaptarse a una variedad de géneros y/o familias vegetales (Rasmussen y Olesen, 2000). Por ello, algunas orquídeas pueden tener un solo polinizador específico como $T$. stramineum que sólo registró a $C$. nitida como su potencial polinizador; mientras que otras especies como Cyrtopodium polyphyllum han registrado hasta tres especies polinizadoras de abejas Centris (Liu y Pemberton, 2010). en la localidad 2 fue la avispa del género Polybia observada en dos ocasiones, posándose en las flores de la orquídea y caminando con movimientos lentos sobre los pétalos y sépalos sin que se diera un contacto con las partes reproductivas, columna estigmática y/o el ginostemo de la flor, por lo que no hubo remoción de los polinios.

La abeja $C$. nitida interactúa con las orquídeas Cyrtopodium polyphyllum y Cyrtopodium punctatum, refiriéndola como polinizadora de $C$. polyphyllum pero no de $C$. punctatum (Liu y Pemberton, 2010). Pemberton (2008) ha observado a $C$. nitida removiendo polinios de Oncidium sphacelatum; mientras que Damon y Cruz (2006) mencionan que $O$. sphacelatum es visitada por Centris trigonoides y Centris mexicana pero al capturar un individuo de $C$. mexicana observaron polinios de $O$. sphacelatum adheridos en la cabeza, refiriéndola como su posible polinizador.

Resulta interesante que entre los pocos registros de $C$. nitida como visitante o polinizador, la abeja pueda presentar un ajustarse morfológico con flores de diferentes orquídeas que difieren en tamaño. Por ejemplo, en C. polyphyllum de flores más grandes, la abeja $C$. nitida puede entrar casi por completo en la cavidad de la flor, adhiriéndose los polinarios en la parte dorsal de su cabeza; mientras que en $T$. stramineum y $O$. sphacelatum de flores más pequeñas, sólo se da el contacto entre la abeja y el ginostemo que contiene a los polinios, adhiriéndose estos en la parte frontal de su cabeza, dándose así la polinización en muchas especies de orquídeas.

Aunque no se descarta que otra especie de abeja Centris esté involucrada en la polinización de $T$. stramineum, como ha sucedido en otras orquídeas del género Trichocentrum, que en una temporada de floración se presenta un polinizador diferente al de otra temporada (Carmona-Díaz y García-Franco, 2009), los cuatro años de observaciones directas e indirectas en las dos localidades de estudio muestran que Centris nitida es el potencial polinizador de esta orquídea.

Revista Científica Biológico Agropecuaria Tuxpan 5 (2) 


\section{CONCLUSIÓN}

Los resultados aquí presentados son los primeros en referir a Centris nitida como el polinizador de $T$. stramineum, por la observación directa y documentada de la remoción y deposición de los polinios de esta orquídea; sin embargo, la falta de información sobre esta orquídea muestra la necesidad de generar cualquier estudio que sirva para conocer de manera más certera, a través de fuentes confiables, aspectos sobre su ecología y biología reproductiva. En futuros estudios resultaría interesante considerar factores que puedan estar implicados en la atracción del polinizador hacia la orquídea, como el tipo de recompensa ofertada, los compuestos aromáticos involucrados en la atracción de la abeja y el comportamiento del polinizador, lo cual permitirá entender más la biología floral y reproductiva de la orquídea y de la interacción Centris-Trichocentrum.

\section{LITERATURA CITADA}

Carmona-Díaz, G. y García-Franco, J. G. 2009. Reproductive success in the Mexican rewardless Oncidium cosymbephorum (Orchidaceae) facilitated by the oil-rewarding Malpighia glabra (Malpighiaceae). Plant Ecology. 203: 253-261. https://doi.org/10.1007/s11258-008-9543-6

Damon, A. A. y Cruz-López, L. 2006. Fragrance in relation to pollination of Oncidium sphacelatum and Trichocentrum oerstedii (Orchidaceae) in the Soconusco Región of Chiapas, México. Selbyana. 27 (2): 186-194.

Flores-Escobar, G., Legaria-Solano, J. P., GilVásquez, I. y Colinas-León, M. T. 2008. Propagación in vitro de Oncidium stramineum Lindl. Una orquídea amenazada y endémica de México. Revista Chapingo Serie Horticultura. 14 (3): 347-353.

https://doi.org/10.5154/r.rchsh.2007.02.009

Goncalves, L., da Silva, C. I. y Tunes, M. L. B. 2012. Collection of Pollen Grains by Centris (Hemisiella) tarsata Smith (Apidae: Centridini): Is C. tarsata an Oligolectic or Polylectic Species? Zoological Studies. 51 (2): 195-203.

Liu, H. y Pemberton, R. 2010. Pollination of an invasive orchid, Cyrtopodium polyphyllum
(Orchidaceae), by an invasive oil collecting bee, Centris nitida, in southern Florida. Botany. 88: 290-295.

https://doi.org/10.1139/B10-017

Moreno-Martínez, D. y Menchaca-García, R. 2009. Trichocentrum stramineum (Bateman ex Lindl.) M.W. Chase y N.H. Williams Especie endémica en peligro. PP: 298-300. En: Gómez-Pompa, A., Thornsten, K. y CastroCortés, R. 2009. Atlas de la Flora de Veracruz. Un patrimonio natural en peligro. Gobierno del estado de Veracruz y Universidad Veracruzana.

Pansarin, E. R. y Pansarin, L. M. 2011. Reproductive biology of Trichocentrum pumilum: an orchid pollinated by oil-collecting bees. Plant Biology. 13: 576-581. https://doi.org/10.1111/j.1438-8677.2010.004

Pemberton, R. W. 2008. Pollination of the ornamental orchid Oncidium sphacelatum by the naturalized oll-collecting bee (Centris nitida) in Florida. Selbyana. 29 (1): 87-91.

Pemberton, R. W. y Liu, H. 2008. Naturalization of the oil collecting bee Centris nitida (Hymenoptera, Apidae, Centrini), a potential pollinator of selected native, ornamental, and invasive plants in Florida. Florida Entomologist. 91 (1): 101-109. https://doi.org/10.1653/0015-4040(2008)091[

Rasmussen, C. y Olesen, J. M. 2000. Oil flowers and oil-collecting bees. Scandinavian Association for Pollination Ecology. 39: 23-31.

Sánchez-Marroquín, H. A. 2017. Diversidad y variación temporal de abejas del género Centris (Hymenoptera: Apidae: Centridini) en el ecosistema de bosque seco Desierto de la Tatacoa (Neiva-Huila). Tesis de Licenciatura. Universidad Militar Nueva Granada. 30 pp.

Soto-Arenas, M. A. y Solano-Gómez, A. R. 2007. Ficha técnica de Trichocentrum stramineum. En:

Tamayo, C. I. 2016. Polinización por engaño de Lophiaris andrewsiae (Orchidaceae: Oncidiinae). Desde el Herbario CICY. 8: 4-8.

Torretta, J. P., Gomiz, N. E., Aliscioni, S. S. y Bello, M. E. 2011. Biología reproductiva de Gomesa Bifolia (Orchidaceae, Cymbidieae, Oncidiinae). Darwiniana. 49 (1): 16-24.

Trejo-Salazar, R. E., Scheinvar, E. y Eguiarte, L. E. 2015. ¿Quién poliniza realmente los agaves? Diversidad de visitantes florales en 3 especies de Agave (Agavoideae: Asparagaceae). Revista Mexicana de Biodiversidad. 86: 358369. https://doi.org/10.1016/j.rmb.2015.0

124

Revista Científica Biológico Agropecuaria Tuxpan 5 (2) 
Vélez-Velandia, E. D. 2012. Revisión del género Centris Fabricius, 1804 (Hymenoptera: Apidae: Centridini) en Colombia. Tesis de Maestría. Facultad de Ciencias. Universidad Nacional de Colombia. Bogotá, Colombia.
$267 \mathrm{pp}$.

Vinson, S. B., Frankie, G. W. y Williams, H. J. 1996. Chemical ecology of bees of the genus Centris (Hymenoptera: Apidae). Florida Entomologist. 79 (2): 109-129.

https://doi.org/10.2307/3495809

Copyright (c) 2017 Saúl Hernández Carm ona, G ustavo Carm ona Diaz, Paulo Cesar Quintana Morales, JorgeE. Morales,

Alejandro Retureta Aponte y Victor Rico Gray

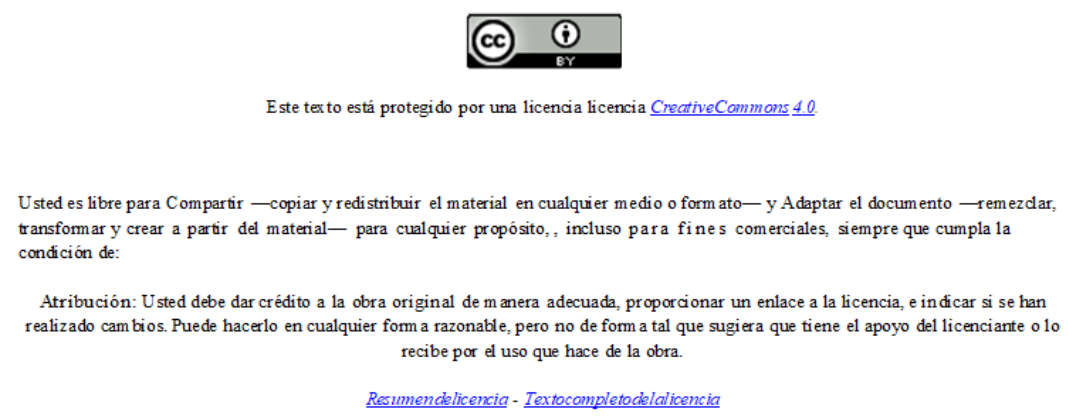

Resumendelicencia - Textocompletodelalicencia 